\title{
and BASIC ASPECTS OF PLAQUE VULNERABILITY
}

T -he treatment of acute coronary syndromes, including acute myocardial infarction and unstable angina, has been one of the most successful contributions in the last 20 years to the improvement of medicine. However, we are still unable to predict them, and therefore to prevent occurrence of acute myocardial infarction and/or death. Major improvements have been made in an effort to understand the mechanism(s) which might lead to acute coronary occlusion: the concept of vulnerable plaque has helped us to understand better the cascade resulting in acute occlusion. Why does a stable plaque become vulnerable, and is it possible to reverse/prevent this process?

The contribution of pathologists has been critical in pointing out the role of the vulnerable plaque in the pathogenesis of acute coronary syndromes, and the major role of thrombus formation. ${ }^{12}$ Initially, plaque rupture has been defined as the mechanism responsible for the evolution from vulnerable plaque to acute occlusion. ${ }^{3}$ Although plaque rupture remains a major cause of plaque complications, other mechanisms have emerged - that is, plaque erosion, and less frequently calcified nodules protruding into the artery lumen. ${ }^{4}$ The fact that coronary thrombosis may occur without plaque rupture is a phenomenon that will have to be taken into account in the definition of vulnerability (table 1 ). These potentially independent mechanisms leading to acute coronary syndromes represent nowadays the new basis to approaching the treatment and prevention of vulnerable plaque. However, this does not eliminate the possibility of other unknown mechanisms undetectable at necropsy. Does a plaque become locally vulnerable or does the patient become vulnerable? The new concept of widespread coronary inflammatory processes during unstable angina has to be integrated into our strategies, provided it is recognised we are not just fighting the culprit vulnerable lesion, but facing a disease involving multiple lesions. ${ }^{5}$ This review will analyse the histologic, cellular, and molecular levels of the putative mechanisms responsible for plaque vulnerability - that is, a plaque prone to acute thrombosis.

\section{VULNERABLE PLAQUE: DEFINITIONS AND MECHANISMS}

Originally the term "vulnerable plaque" was specifically used to define a plaque prone to rupture. We have learned a great deal from pathologists about the histological aspects of the plaque in patients who present with sudden cardiac death. ${ }^{6}$ Three situations have been described: plaque rupture, plaque erosion, and plaque calcification ${ }^{4}$ (table 1 ). Since plaque rupture is not the exclusive histologic situation resulting in thrombosis, we therefore propose to extend the definition of the vulnerable plaque to "a plaque prone to thrombose", resulting in acute coronary syndromes including acute myocardial infarction or unstable angina via permanent or transitory occlusion. Thus, the concept of plaque vulnerability has to be associated with the occurrence of thrombus formation. We would complete the histopathological approach by a hypothesis: vulnerable plaque is the resulting process of the lack of healing at the site of atherogenesis. We therefore propose a fourth situation which occurs after therapeutic strategies aimed at inhibiting plaque healing in conditions where the therapy has exceeded its goal, resulting in late occlusions secondary to brachytherapy or drug eluting stents targeted against the cell cycle.

\section{PLAQUE RUPTURE}

Davies and colleagues described plaque rupture almost 30 years ago. ${ }^{1}$ It is the most popular concept of vulnerable plaque although it should not be considered as the only one. Its occurrence varies from $60-75 \%$. It has been generally considered as the standard model: the plaque prone to rupture is made of a large lipid core composed of foam cells, apoptotic and necrotic cells, and debris, and is separated from the lumen by a fibrous cap (mainly comprising collagen, proteoglycans, and smooth muscle cells) which is actively weakened both by a lytic process and a lack of repair; this results eventually in the plaque fissuring at one point, which ultimately brings the platelets into contact with the content of the lipid core, and the blood coagulation factors together with tissue factor (fig 1). Thus, the thinner the fibrous cap, the higher the risk of rupture. These criteria have been clearly established. The so-called "plaque rupture" has been extensively considered by pathologists 
Table 1 Modified American Heart Association classification based on morphological description

\begin{tabular}{|c|c|c|}
\hline & Description & Thrombosis \\
\hline $\begin{array}{l}\text { Non-atherosclerotic intimal lesions } \\
\text { Intimal thickening }\end{array}$ & $\begin{array}{l}\text { The normal accumulation of smooth muscle cells (SMCs) in the intima in the } \\
\text { absence of lipid or macrophage foam cells }\end{array}$ & Absent \\
\hline Intimal xanthoma, or "fatty streak" & $\begin{array}{l}\text { Luminal accumulation of foam cells without a necrotic core or fibrous cap. } \\
\text { Based on animal and human data, such lesions usually regress. }\end{array}$ & Absent \\
\hline \multicolumn{3}{|l|}{ Progressive atherosclerotic lesions } \\
\hline Pathological intimal thickening & $\begin{array}{l}\text { SMCs in a proteoglycan-rich matrix with areas of extracellular lipid } \\
\text { accumulation without necrosis }\end{array}$ & Absent \\
\hline Erosion & Luminal thrombosis; plaque same as above & $\begin{array}{l}\text { Thrombus mostly mural and } \\
\text { infrequently occlusive }\end{array}$ \\
\hline Fibrous cap atheroma & Well formed necrotic core with an overlying fibrous cap & Absent \\
\hline Erosion & $\begin{array}{l}\text { Luminal thrombosis; plaque same as above; no communication of thrombus } \\
\text { with necrotic core }\end{array}$ & $\begin{array}{l}\text { Thrombus mostly mural and } \\
\text { infrequently occlusive }\end{array}$ \\
\hline Thin fibrous cap atheroma & $\begin{array}{l}\text { A thin fibrous cap infiltrated by macrophages and lymphocytes with rare } \\
\text { SMCs and an underlying necrotic core }\end{array}$ & $\begin{array}{l}\text { Absent; may contain intraplaque } \\
\text { haemorrhage/fibrin }\end{array}$ \\
\hline Plaque rupture & $\begin{array}{l}\text { Fibroatheroma with cap disruption; luminal thrombus communicates with the } \\
\text { underlying necrotic core }\end{array}$ & Thrombus usually occlusive \\
\hline Calcified nodule & Eruptive nodular calcification with underlying fibrocalcific plaque & Thrombus usually non-occlusive \\
\hline Fibrocalcific plaque & $\begin{array}{l}\text { Collagen-rich plaque with significant stenosis usually contains large areas of } \\
\text { calcification with few inflammatory cells; a necrotic core may be present }\end{array}$ & Absent \\
\hline
\end{tabular}

as the process underlying thrombus formation. Many strategies have been tested in order to inhibit plaque rupture and thrombus formation, in particular the statins because of their numerous pleiotropic properties. ${ }^{7}$

\section{Cells involved in plaque rupture}

At the cellular level, the principal players are the endothelial cells, smooth muscle cells, macrophages, T lymphocytes, and neutrophils, and their mutual interactions will result in plaque rupture.

Endothelial cells play a pivotal role since they represent the major barrier against thrombosis, and their dysfunction results in attracting inflammatory cells (dysfunctional endothelial cells allow $\mathrm{T}$ lymphocyte recruitment via overexpression of the cell adhesion molecules) within the plaque where they can induce spasm, and participate in the thrombogenesis. The smooth muscle cells are responsible for extracellular matrix production. In contrast to the classical concept implying their pathogenic role in atherogenesis, smooth muscle cells guarantee the integrity of the artery wall: it is not surprising that they have been shown to be decreased, apoptotic, and not able to warrant the synthesis and tissue repair of the extracellular matrix, which is actively destroyed by the macrophages in plaque rupture. ${ }^{8}$ Macrophages represent the "Trojan horse" of the atherosclerotic plaque. They play a central role in non-specific inflammation by secreting cytokines, and they concentrate the peroxidised lipids to become foam cells. Foam cells induce expression of the cell adhesion molecules in endothelial cells. Moreover, they may actively weaken the fibrous cap by secreting matrix metalloproteinases (MMPs). Macrophages in apoptosis are more abundant in the ruptured fibrous cap, as compared to stable plaques or unruptured fibrous caps. ${ }^{9}$ Inflammation is further developed by the activated macrophages which secrete cytokines (tumour necrosis factor $\alpha$, interleukin (IL)-1, IL-4). Neutrophils are present both in blood and in the plaque, secrete MMP 8, and actively contribute to the inflammatory process. Although the number of neutrophils infiltrated in human atherectomy specimens is equivalent between plaque rupture and plaque erosion, there are more activated neutrophils (neutral endopeptidase positive) in plaque rupture. ${ }^{10} \mathrm{~T}$ lymphocytes are responsible for the specific inflammation, depending on epitopes, namely oxidised low density lipoprotein (LDL). $\mathrm{T}$ lymphocytes transmit messages to smooth muscle cells and macrophages and influence the inflammatory process, particularly via the activation of MMP.

\section{Mechanisms of plaque "vulnerabilisation"}

Mechanisms of plaque rupture have been extensively studied and several parameters have been found to interact: the extracellular matrix, the inflammatory cells, the lipid core, and more particularly the oxidised lipids, and the factors inducing thrombosis.

The concept of plaque rupture is based on the weakening of the fibrous cap which protects the blood from the thrombogenic lipid core. Libby's group has played a leading role in the identification of the mechanisms of plaque rupture. ${ }^{2}{ }^{11-13}$ They have demonstrated that the extracellular matrix is actively destroyed by MMPs which are locally overexpressed by the macrophages. ${ }^{11}$ In the weakened plaque the number of macrophages producing MMP is increased and the number of smooth muscle cells repairing the extracellular matrix is decreased. This imbalance between extracellular matrix synthesis and degradation is a solid basis for plaque rupture. Activated $\mathrm{T}$ lymphocytes produce interferon (which decreases the synthesis of collagen I and III by smooth muscle cells ${ }^{12}$ ). The $\mathrm{T}$ lymphocytes also influence the degradation of the extracellular matrix by stimulating macrophage production of MMP involving CD40 ligation. ${ }^{13}$

MMP expression is also induced by oxidised lipids contained in the lipid core. Oxidised LDL contributes to the plaque weakening by decreasing the natural inhibitor of MMP 


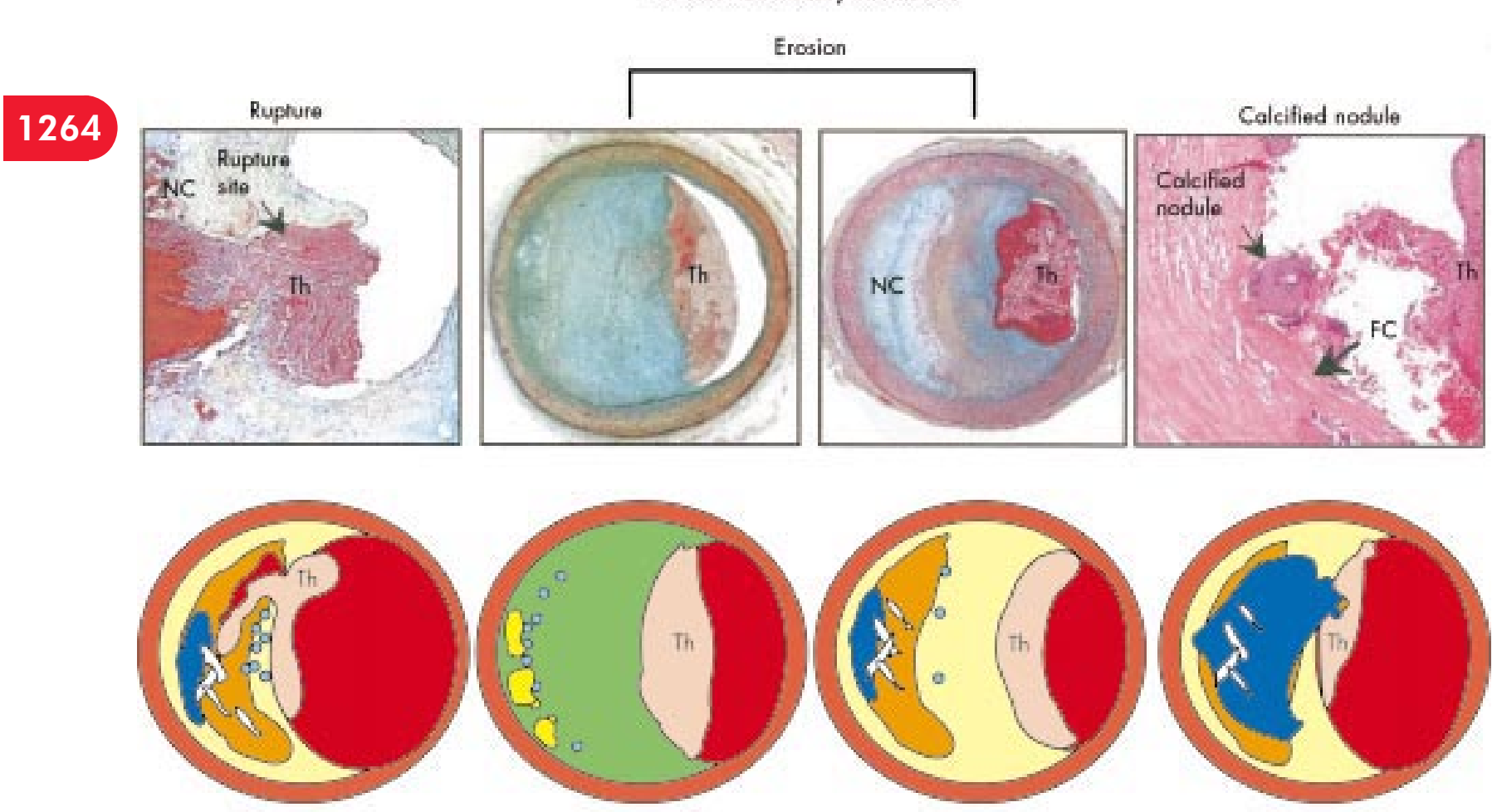

Figure 1 Atherosclerotic lesions with luminal thrombi. Ruptured plaques are thin fibrous cap atheromas with luminal thrombi (Th). These lesions usually have an extensive necrotic core $(\mathrm{NC})$ containing large numbers of cholesterol crystals and a thin fibrous cap (<65 $\mu \mathrm{m})$ infiltrated by foamy macrophages and a paucity of $T$ lymphocytes. The fibrous cap is thinnest at the site of rupture and consists of a few collagen bundles and rare smooth muscle cells. The luminal thrombus is in communication with the lipid-rich necrotic core. Erosions occur over lesions rich in smooth muscle cells and proteoglycans. Luminal thrombi overlie areas lacking surface endothelium. The deep intima of the eroded plaque often shows extracellular lipid pools, but necrotic cores are uncommon; when present, the necrotic core does not communicate with the luminal thrombus. Inflammatory infiltrate is usually absent, but if present, is sparse and consists of macrophages and lymphocytes. Calcified nodules are plaques with luminal thrombi showing calcific nodules protruding into the lumen through a disrupted thin fibrous cap (FC). There is absence of an endothelium at the site of the thrombus, and inflammatory cells (macrophages, T lymphocytes) are absent. Reproduced from Virmani et al, ${ }^{4}$ with permission.

(TIMP-1) expressed by the macrophages, and by inducing apoptosis of the smooth muscle cells. In contrast, high density lipoprotein (HDL) has a protective effect. Paraoxonase and lecithine-cholesterol acyltransferase (LCAT) are HDL associated enzymes; paraoxonase protects LDL and HDL from oxidation, and LCAT prevents the formation of oxidised lipids in LDL. More generally oxidant stress may play a key role in increasing the vulnerability of the plaque; in the model of apoE and L-gulonolactone oxidase deficient mice (dependent on dietary vitamin $\mathrm{C}$ ), the chronic deficiency of vitamin $\mathrm{C}$ clearly alters collagen deposition resulting in a fascinating model of vulnerable plaque prone to rupture (that is, a thinner fibrous cap and a lipid core devoid of collagen ${ }^{14}$ ).

More recently, it has been proposed that the phenomenon of plaque rupture might occur at several sites rather than an isolated one. This has been illustrated by the concept of widespread activation of neutrophils (measured by the concentration of neutrophil myeloperoxidase in blood) proposed by Maseri's group, as they traverse the whole coronary vasculature of patients presenting with unstable angina. ${ }^{5}$ Similarly, angioscopy in patients with myocardial infarction detected that the three coronary arteries were widely diseased, with yellow plaques, and thrombus was present in the culprit artery. Systematic evaluation of the three coronary arteries by intracoronary ultrasound revealed that a clinically active ruptured plaque was accompanied by multiple plaque ruptures in $79 \%$ of cases. This shows that there is likely a strong interaction between the local inflammatory process and the systemic inflammatory process. Whether one process is triggering the other remains unknown. Practically, the concept of plaque vulnerability should not let us underestimate the potential vulnerability of the patient himself-that is, an identified vulnerable plaque may be only one among several factors that can be "switched on". This should be borne in mind if we limit our therapeutic strategy to a local action, which gives preference to the glycoprotein IIb/IIIa inhibitors.

It has been postulated that infectious agents like Chlamydia pneumoniae could induce atherosclerotic lesions. However, C pneumoniae alone repeatedly failed to generate an atherosclerotic plaque. C pneumoniae is present in the foam cells since it is carried by the monocytes-macrophages that penetrate the plaque. Nevertheless, it can be proposed that an infectious disease may non-specifically activate a stable plaque via the inflammatory process that is accompanying the disease itself. 
Table 2 Risk factors associated with plaque rupture or erosion

\begin{tabular}{|c|c|c|c|c|c|c|}
\hline $\begin{array}{l}\text { Age } \\
\text { (years) }\end{array}$ & $\begin{array}{l}\text { High } \\
\text { cholesterol }\end{array}$ & Smoker & Sex & $\begin{array}{l}\text { Plaque } \\
\text { rupture }\end{array}$ & $\begin{array}{l}\text { Plaque } \\
\text { erosion }\end{array}$ & Reference \\
\hline$>50$ & 270 & $33 \%$ & Female & + & - & Burke et $a l^{15}$ \\
\hline$<50$ & 194 & $78 \%$ & Female & - & + & Burke et $a l^{15}$ \\
\hline- & + & $41 \%$ & Male & + & - & Burke et $a^{k}$ \\
\hline 47.9 & - & $75 \%$ & Male & - & + & Burke et a ${ }^{k}$ \\
\hline 30.9 & $50 \%$ & $0 \%$ & Male & + & - & Henriquez et $\left.a\right|^{18}$ \\
\hline 30.9 & $22 \%$ & $44 \%$ & $44 \%$ female & - & + & Henriquez et $a l^{18}$ \\
\hline- & - & - & Female & $38 \%$ & $62 \%$ & Arbustini et $a^{16}$ \\
\hline- & - & - & Male & $82 \%$ & $18 \%$ & Arbustini ${ }^{16}$ \\
\hline
\end{tabular}

Mechanical aspects of plaque rupture also have to be taken into account. The heterogeneity of the unstable plaque, the permanent stress caused by the physiological pressure changes, the pathological pressure elevations, and spasm all favour rupture. By finite element analysis, it has been shown that the mechanical properties of the fibrous cap plays an important role in plaque rupture, and more particularly the cap thickness. Moreover, the changes in the constituents of the fibrous cap may explain the rupture: a decrease in collagen but also in proteoglycans may modify the mechanical properties of the fibrous cap. Thus, failure to compensate for cap fatigue caused by cell death and matrix loss may contribute to plaque rupture.

\section{PLAQUE EROSION}

Plaque erosion is a less known process which has been identified by Virmani's group ${ }^{4915}$ and confirmed by Arbustini and colleagues. ${ }^{16}$ Its importance has been less emphasised although it provides a critical message: it seems to concern a distinct group of patients (young, smokers, females), and there is increasing evidence that vulnerable plaque may not represent a unique disease, but is the result of different causes including plaque rupture and plaque erosion. It consists of the association of a thrombus with erosion of the endothelial cells at the site of occlusion with a lack of plaque rupture (fig l). In contrast with the ruptured plaque, the eroded plaque is rich in smooth muscle cells and proteoglycans, and contains fewer macrophages and Tlymphocytes, and fewer calcifications. The rate of plaque erosion in necropsy studies of patients after sudden coronary death was 25-44\%. ${ }^{6}{ }^{15}{ }^{17}$ Therefore plaque erosion should not be ignored when it comes to developing prevention strategies for vulnerable plaque. More recently, Becker's group studied the histology of 11 patients younger than 35 years old who presented with sudden cardiac death. ${ }^{18}$ Nine (four female) had plaque erosion (82\%). Only one had a high cholesterol value, and four were smokers. This clearly defines plaque erosion as an entity which may be independent of plaque rupture (table 2). Interestingly, inflammation does not appear to play as large a role in plaque erosion as it does in plaque rupture.

\section{Mechanisms of plaque erosion " vulnerability"}

Plaque erosion is defined as an abrasion of the endothelium without plaque rupture. The problem is to know how endothelial cells disappear, and what are the circumstances which favour this erosion: does it come from the endothelium, the wall, or the blood? Apoptosis of the endothelium has been recently shown by Mallat and colleagues, leading to acute coronary syndromes. ${ }^{19}$ Apoptosis results in plaque erosion. It is well known that endothelial dysfunction is the initial event of atherogenesis. Part of the dysfunction results in loss of nitric oxide (NO) bioavailability. Among the mechanisms of endothelial protection provided by NO are the inhibition of platelet aggregation, of spasm, and particularly of endothelial apoptosis. Reduction of NO in atherosclerotic lesions impairs the antithrombotic and antispasm protection. Thus, endothelial apoptosis may be a rationale for plaque erosion.

Are there triggers of endothelial apoptosis? It has been shown that all risk factors for atherosclerotic disease promote endothelial dysfunction and apoptosis in vitro. However, in vivo, Virmani and colleagues demonstrated that smoking was related to plaque erosion, whereas high cholesterol was surprisingly not a risk factor. ${ }^{15}{ }^{17}$ They also clearly showed that the population at risk for plaque erosion was younger than the population with plaque rupture, and comprised more women. Interestingly, women with plaque rupture are less numerous. Does this mean that there is a risk of plaque erosion for nonmenopausal females? Is oestrogen a higher risk factor and do oral contraceptives favour endothelial cell apoptosis?

How does apoptosis lead to plaque erosion? It is known that endothelial progenitor cells repair the endothelium, and that endothelial progenitor cells are altered in patients with risk factors. ${ }^{20}$ Dimmeler and colleagues hypothesise that the endothelial repair may thus be impaired in those patients who undergo plaque erosion. ${ }^{21}$ Apoptotic cells result in microparticles which are raised in patients with acute coronary syndromes and are prothrombogenic. ${ }^{19}$ This gives a basis for acute thrombosis in patients with plaque erosion secondary to severe endothelial cell apoptosis. However, the triggers of endothelial cell apoptosis remain vague, whereas the consequences of plaque erosion may be catastrophic. The problem is that there is no proof yet that preventing endothelial cell apoptosis will be sufficient to control plaque erosion, and prevention strategies remain to be defined, even if the population at risk is grossly defined. The mechanisms of regulation of endothelial cell apoptosis to be targeted involve the cellmatrix interaction.

Like plaque rupture, plaque erosion may in part be caused by a mechanical process. Indeed it can be hypothesised that repeated spasm at the same site provoked by smoking may alter endothelial cells, thereby facilitating endothelial detachment from the artery wall. Moreover, flow turbulence may increase endothelial apoptosis, and facilitate plaque erosion. It has been shown that shear stress could prevent apoptosis by stimulating the production of low NO concentrations in endothelial cells via increased gene expression of NO synthase. However, we do not know whether plaque erosion exists without thrombosis. We previously showed in the New Zealand white rabbit after arterial air desiccation that both thrombosis and endothelial dysfunction were induced at the site of an artery segment filled with an adenoviral solution. ${ }^{22}$ 


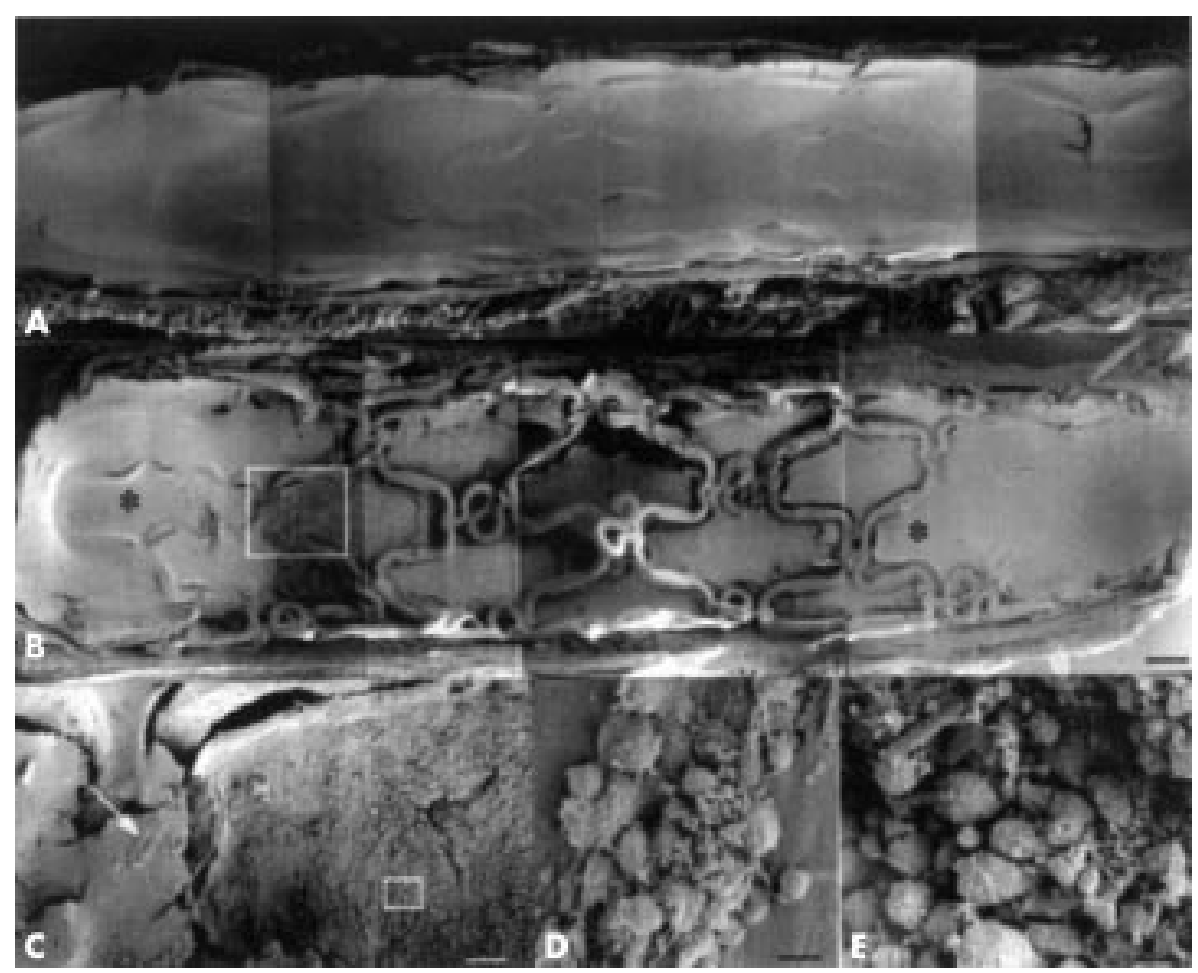

Figure 2 Low power scanning electron micrograph of control stent (A) and $48 \mu \mathrm{Ci}$ stent (B) at 12 months. The entire control stent is endothelialised, whereas the $48 \mu \mathrm{Ci}$ stent is endothelialised at the ends (* in B). Parts of the central portion of the $48 \mu \mathrm{Ci}$ stent are non-endothelialised, and bare or partially covered struts are present. The transition zone between endothelialisation and non-endothelialisation is outlined in $B$ and shown at medium power in $C$. Two areas are further magnified larrow and box in $\mathrm{C}$ corresponding to $D$ and $E$, respectively) to show adherent platelets and inflammatory cells on partially endothelialised (D) and non-endothelialised $(E)$ parts of the stent. Bars $=0.5 \mathrm{~mm}$ in $A$ and $B$, $0.1 \mathrm{~mm}$ in $C, 0.01 \mathrm{~mm}$ in $\mathrm{D}$ and $\mathrm{E}$. Reproduced from: Farb A, et al. Circulation 2001;103:1912-9, with permission of the American Heart Association.

This could be interpreted as an infectious trigger resulting non-specifically in arterial vulnerability in a model of plaque erosion.

\section{PLAQUE CALCIFICATION}

Plaque calcification was also described by Virmani and colleagues as a lesion associated with coronary thrombosis in the absence of eroded or ruptured plaque, although more seldom (5\%). ${ }^{4}$ The calcified nodules protrude into the lumen and are not associated with the lipid core (fig 1). There are no data available regarding the onset of this presentation.

\section{POST-TREATMENT ARTERIAL THROMBOSIS}

We have learnt from the two leading causes of thrombosiserosion and ruptured plaques - that cell death (smooth muscle or endothelial) is a critical step. More generally, lack of healing results in thrombosis, namely by lack of endothelial regeneration and/or endothelial dysfunction, and lack of fibrous cap repair by smooth muscle cells. Thus, strategies aimed at inhibiting the healing process, with the goal of preventing restenosis after angioplasty, carry the risk of favouring thrombosis. Indeed, brachytherapy and coated stents with molecules acting on the cell cycle have shown the occurrence of late thrombosis. The treated lesion exhibits an atherosclerotic plaque with neointimal necrosis, cell death in experimental models, and "black holes" — that is, loss of neointima behind the cell struts in humans detected by intracoronary ultrasound. Indeed, the healing process which routinely covers the struts of a bare stent is impaired by active coated stents targeted against cell proliferation, thereby inhibiting endothelial coating and extracellular matrix repair, whereas restenosis is inhibited (fig 2).

\section{THROMBOSIS}

Thrombus occurrence is the obligatory signature of a complicated vulnerable plaque. The thrombus formation may occur from elements issued from the plaque itself and/or the interaction between blood and activated platelets and dysfunctional endothelium. Several plaque components may be either directly or indirectly thrombogenic. Macrophage foam cells overexpress tissue factor. ${ }^{23}$ Tissue factor is a coagulation protein (via interaction with factor VII) that is secreted by the endothelium and macrophages, and is present in the plaque and in inflammatory conditions. It plays an important role in the thrombotic process. T lymphocytes induce tissue factor from macrophages. T lymphocytes, via the CD40 ligand, activate the macrophages and more particularly secretion of the tissue factor. ${ }^{13}$ Tissue factor secretion is also increased by oxidised LDL via the endothelial cells and smooth muscle cells. In vitro, oxidised LDL increases plasminogen activator inhibitor (PAI)-1, and decreases protein $\mathrm{C}$ and tissue plasminogen activator (t-PA) in endothelial cells. Lipoprotein Lp(a) participates in the promotion of thrombosis by interfering with plasminogen and overexpressing PAI-1. Increased concentrations of PAI- 1 and apo(a) have been found in atherectomy samples of patients presenting with acute coronary syndromes; interestingly there was a correlation between concentrations of PAI- 1 and apo(a) and macrophage density. ${ }^{24}$

Blood also plays a role in the occurrence of local thrombus formation. Platelet activation is prompted by collagen exposure from the subendothelium: this represents a simple substratum of acute thrombosis in the setting of plaque erosion. Inhibition of platelet activation is reduced in unstable plaque by reduction of prostacyclin. The nature of the plaque itself may also participate in the thrombus formation. CD44 and proteoglycans, namely versican and hyaluronan, were found in high concentrations in eroded plaques. ${ }^{25}$ It has been suggested that hyaluronan could be involved in the development of thrombosis. CD44 receptors mediate platelet adhesion to hyaluronan. Hyaluronan also increases fibrin formation. Together these elements may facilitate the occurrence of thrombus in eroded plaques. 


\section{CONCLUSIONS}

The vulnerable plaque is a challenging, vexing topic. Pathologists have been most helpful in classifying the lesions. A basic approach with appropriate models is clearly necessary to address in vivo the issues regarding the mechanisms and treatment. It appears that rupture or erosion of the plaque are the leading causes of death, but we do not know whether other potential mechanism(s) may result in thrombus formation in vivo. Moreover, at our current level of knowledge, we are not able to declare any part of the arterial tree invulnerable. The concept of a vulnerable patient is growing over the concept of vulnerable plaque.

\section{REFERENCES}

1 Davies MJ, Thomas A. A thrombosis and acute coronary-artery lesions in sudden cardiac ischemic death. N Engl J Med 1984;310:1 137-40.

- This study is one of the earliest to suggest a link between plaques and coronary thrombosis.

2 Libby P. Molecular bases of the acute coronary syndromes. Circulation 1995;91:2844-50.

- This is a very complete review on plaque rupture, illustrated by world recognised figures to support the role of plaque rupture.

3 Falk E. Why do plaques rupture? Circulation 1992;86(6 supp):III30-42.

- This paper was one of the earliest presentations of plaque rupture.

4 Virmani R, Kolodgie FD, Burke AP, et al. Lessons from sudden coronary death: a comprehensive morphological classification scheme for atherosclerotic lesions. Arterioscler Thromb Vasc Biol 2000;20:1262-75.

- This work is critical since it carefully explains why the old classification was unsatisfactory, and provides a modified classification.

5 Buffon A, Biasucci LM, Liuzzo G, et al. Widespread coronary inflammation in unstable angina. N Engl J Med 2002;347:5-12.

- This elegant study carefully demonstrates the concept of patient vulnerability.

6 Burke AP, Farb A, Malcom GT, et al. Coronary risk factors and plaque morphology in men with coronary disease who died suddenly. $N$ Engl J Med 1997;336:1276-82.

- The authors clearly highlight the risk factors of men associated with vulnerable plaque.

7 Aikawa M, Rabkin E, Okada Y, et al. Lipid lowering by diet reduces matrix metalloproteinase activity and increases collagen content of rabbit atheroma: a potential mechanism of lesion stabilization. Circulation 1998;97:2433-44.

- In this study, the authors demonstrate how statins are effective in stabilising plaque via MMP activity.

8 Lafont A, Libby P. The smooth muscle cell: sinner or saint in restenosis and the acute coronary syndromes? J Am Coll Cardiol 1998;32:283-5.

9 Kolodgie FD, Narula J, Burke AP, et al. Localization of apoptotic macrophages at the site of plaque rupture in sudden coronary death. Am J Pathol 2000; 157: 1259-68
10 Naruko T, Ueda M, Haze K, et al. Neutrophil infiltration of culprit lesions in acute coronary syndromes. Circulation 2002; 106:2894-900.

11 Galis ZS, Sukhova GK, Lark MW, et al. Increased expression of matrix metalloproteinases and matrix degrading activity in vulnerable regions of human atherosclerotic plaques. J Clin Invest 1994:94:2493-503.

- In this study, the authors demonstrate very clearly the role of MMP in plaque rupture.

12 Amento EP, Ehsani N, Palmer H, et al. Cytokines and growth factors positively and negatively regulate interstitial collagen gene expression in human vascular smooth muscle cells. Arterioscler Thromb $1991 ; 11: 1223-30$

13 Mach F, Schonbeck U, Bonnefoy JY, et al. Activation of monocyte/macrophage functions related to acute atheroma complication by ligation of CD40: induction of collagenase, stromelysin, and tissue factor. Circulation 1997:96:396-9.

14 Nakata Y, Maeda N. Vulnerable atherosclerotic plaque morphology in apolipoprotein E-deficient mice unable to make ascorbic acid. Circulation 2002; 105: 1485-90.

15 Burke AP, Farb A, Malcom GT, et al. Effect of risk factors on the mechanism of acute thrombosis and sudden coronary death in women. Circulation 1998;97:2110-6

16 Arbustini E, Dal Bello B, Morbini P, et al. Plaque erosion is a major substrate for coronary thrombosis in acute myocardial infarction. Heart 1999;82:269-72.

17 Burke AP, Farb A, Malcom G, et al. Effect of menopause on plaque morphologic characteristics in coronary atherosclerosis. Am Heart J $2001 ; 141$ (2 suppl):S58-62

18 Henriques de Gouveia $\mathbf{R}$, van der Wal AC, van der Loos CM, et al Sudden unexpected death in young adults. Discrepancies between initiation of acute plaque complications and the onset of acute coronary death. Eur Heart J 2002; 23: 1433-40.

- This excellent study shows that in a population of young patients the aetiology of vulnerability is clearly plaque erosion. Moreover, it shows that there is no relation between cholesterol disorders and plaque erosion.

19 Mallat Z, Hugel B, Ohan J, et al. Shed membrane microparticles with procoagulant potential in human atherosclerotic plaques: a role for apoptosis in plaque thrombogenicity. Circulation 1999;99:348-53.

20 Vasa M, Fichtlscherer S, Aicher A, et al. Number and migratory activity of circulating endothelial progenitor cells inversely correlate with risk factors for coronary artery disease. Circ Res 2001;89:E1-7.

21 Dimmeler S, Haendeler J, Zeiher AM. Regulation of endothelial cell apoptosis in atherothrombosis. Curr Opin Lipidol 2002;13:531-6.

This is a very original review shedding light on the relation between endothelial apoptosis and plaque erosion.

22 Lafont A, Durand E, Vilde F, et al. Thrombus generation after adenovirus-mediated gene transfer into atherosclerotic arteries. Hum Gene Ther 1998;9:2795-800.

23 Shindo J, Ishibashi T, Kijima M, et al. Increased plasminogen activator inhibitor- 1 and apolipoprotein (a) in coronary atherectomy specimens in acute coronary syndromes. Coron Artery Dis 2001;12:573-9.

24 Wilcox JN, Smith KM, Schwartz SM, et al. Localization of tissue factor in the normal vessel wall and in the atherosclerotic plaque. Proc Natl Acad Sci U S A 1989:86:2839-43.

25 Kolodgie FD, Burke AP, Farb A, et al. Differential accumulation of proteoglycans and hyaluronan in culprit lesions: insights into plaque erosion. Arterioscler Thromb Vasc Biol 2002;22:1642-8. 